\title{
Link Quality Assessment Algorithm for Heterogeneous Self-organizing Maritime Communications Network
}

\author{
Krzysztof Bronk, Adam Lipka, and Rafał Niski \\ National Institute of Telecommunications, Wireless Systems and Networks Department, Gdańsk, Poland
}

https://doi.org/10.26636/jtit.2018.121217

\begin{abstract}
The article introduces a method of performing a radio link quality assessment based on the Link Quality Indicator (LQI) which will be calculated for every system that is available. The method presented has been developed during the netBaltic project completed in Poland and generally applies to the so-called maritime zone A, i.e. the sea area where ships are still within the range of shore-based radio communication systems, particularly 3G/LTE cellular networks. The algorithm was developed based on the results of measurements obtained during two separate campaigns. That measurement data served as a basis for the method's initial assumptions and was utilized during the method's verification.
\end{abstract}

Keywords-3G, cellular systems, link quality indicator, LTE, maritime radiocommunications.

\section{Introduction}

The main purpose of the netBaltic project is to develop a multi-system, self-organizing wideband maritime communications network which will support modern e-navigation features and improve the overall safety of maritime navigation. The network will offer fast data transmission between vessels, vessels and on-shore infrastructure, as well as between vessels and the Internet, using the most suitable link selected from among those available. For the purpose of the project, it is assumed that the entire sea area is divided into three separate zones to which various communication mechanisms apply. That arrangement is illustrated in Fig. 1 [1]:

- in zone A, ships are close enough to the coast to be within range of the shore-based communication systems (mostly cellular networks) and can utilize such systems to communicate with one another (the following paper applies only to zone A),

- in zone B, ships can only communicate with the shore via other ships that are within zone A,

- in zone C, ships are out of any network's range and the main option in this case is a buffered transmission (ship-to-ship communication is also possible, however).
One of the major tasks performed in the course of the netBaltic project is the development of an algorithm enabling to assess quality of the wireless communication links available. The analysis of the links' quality and stability is undoubtedly an important issue and, as such, it is frequently addressed in projects and publications related to contemporary radio communications [2], [3]. The mechanism introduced here will enable the selection of the most suitable (optimal) wireless communication link for the specific type of service. The link's quality will be evaluated based on the so-called Link Quality Indicator (LQI) metric, whose value will be calculated for every system available. The autonomous analysis functionality enabling to assess the quality of various communication links, as well as seamless roaming between them are some of the most important features of the netBaltic network.

The network switches between various communications standards/systems and operators to provide the best quality of the connection and to avoid - to the extent possible expensive satellite links.

The link quality assessment concept presented in this paper was developed following a thorough analysis of the measurement data obtained during two campaigns that were performed on the Baltic Sea in the course of the netBaltic project. The first took place in the summer of 2015 and its scope mostly included range and quality measurements of the selected cellular systems. As a result, almost 15,000 measurement points were obtained for LTE networks, and roughly 5,000 measurement points for 3G. The results of this campaign helped define the initial assumptions for the LQI algorithm and were utilized during preliminary verification of the method.

The second campaign was held in the winter of 2017 and its main purpose was to gather more detailed results regarding LTE and 3G networks under maritime conditions. This time, the measurement points were obtained with a much greater frequency and, as a result, nearly 30,000 points were collected for LTE and over 42,000 points for 3G networks. These results were sufficient to introduce some modifications into the original version of the LQI algorithm and were later used during its second and final verification. 

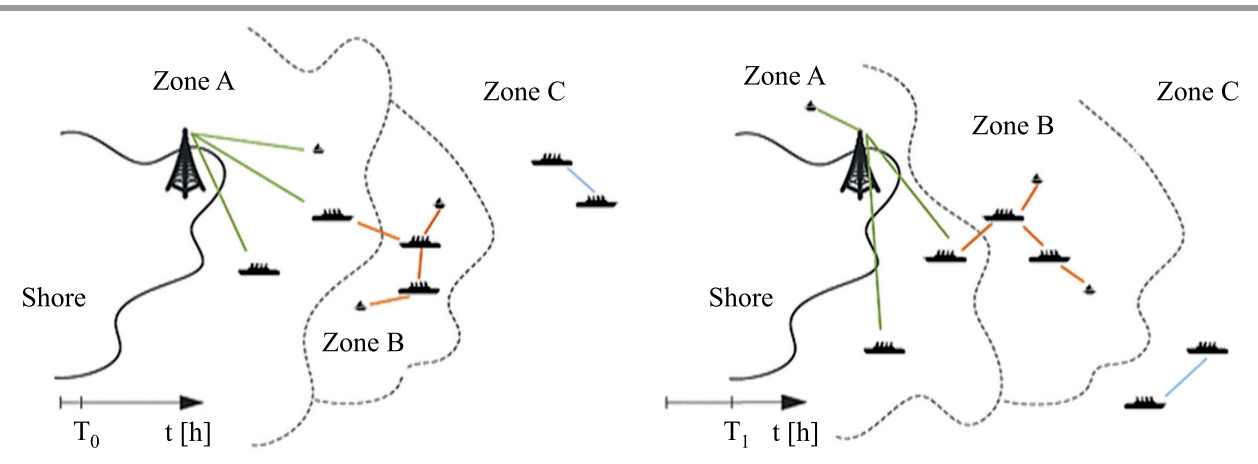

Fig. 1. Operating principle of zones A, B and C introduced for the purpose of the netBaltic project.

\section{Measurement Methodology}

As it was previously mentioned, the results of the measurement campaigns have been relied upon to define the LQI algorithm. Below, we will provide some explanations concerning the methodology used.

Both measurements were performed on a ship-to-shore basis, meaning that the ship participating in the campaign maintained communications with the infrastructure of shore-based cellular systems (i.e. base stations). No shipto-ship communication was tested at this stage.

The main component utilized during the campaigns was a Mobile Measurement Platform [4] which was developed by the National Institute of Telecommunications to facilitate measurements of various quality-related parameters of radio communications systems (data rate, delays, received signal level, etc.). Its general architecture is depicted in Fig. 2.

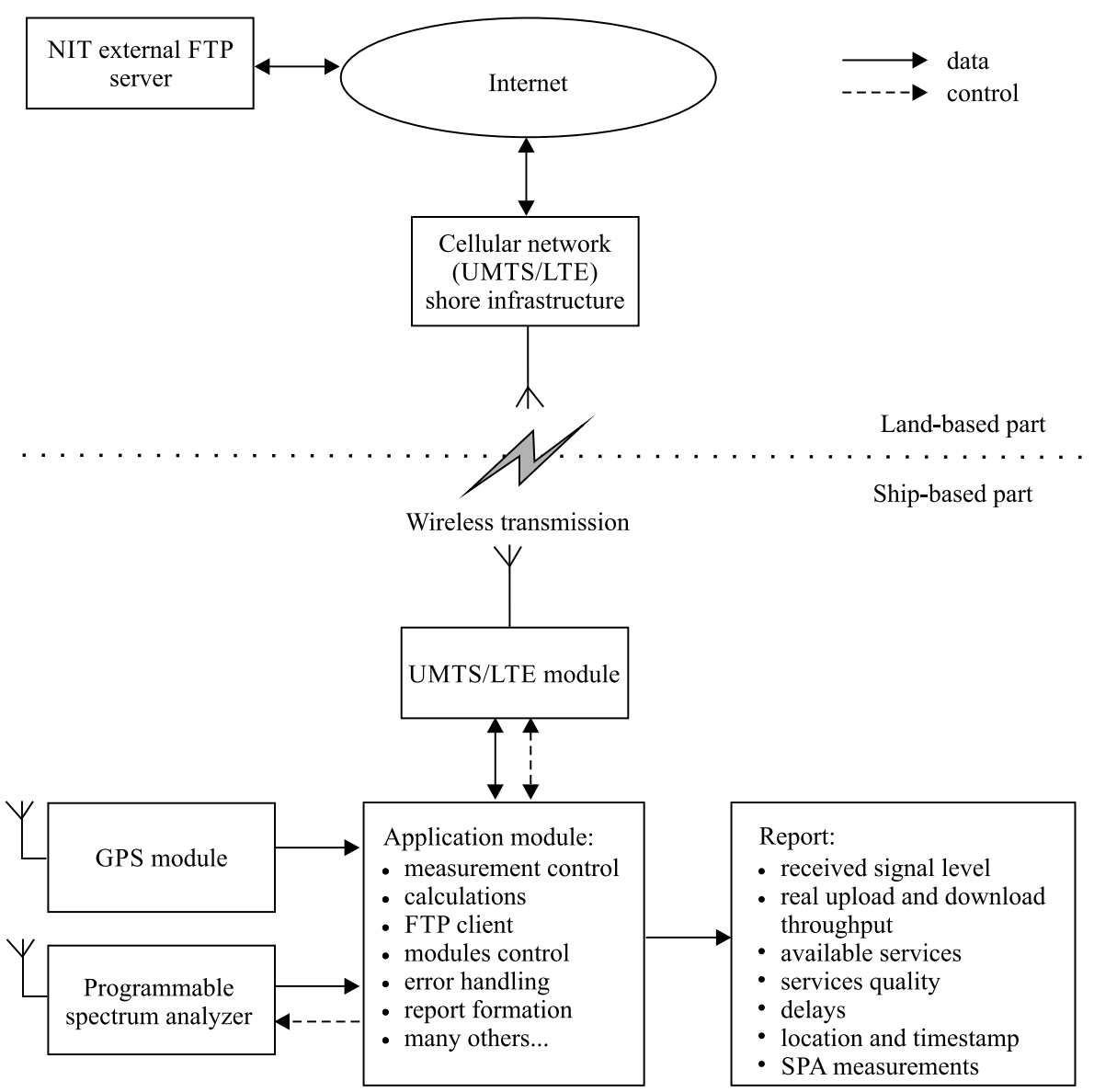

Fig. 2. Mobile Measurement Platform architecture. 
Using the $3 \mathrm{G} /$ LTE module ${ }^{1}$, a radio link between the ship and an external FTP server was set up. Having established the connection, it was possible to initiate a bi-directional data transmission between FTP and the ship, and to measure its duration. To test the downlink transmission, the application module started to download a file from the FTP server. To test the uplink transmission, another file was uploaded into the FTP. The size of the file that was being transmitted at a given moment was not random, but it was selected by the application module using a special algorithm. If the available throughput was low, the file size was gradually decreased to avoid excessive duration of a single measurement. If the throughput increased, it was also possible to increase the test file size. So, we might say that in this case the file size was being changed adaptively. After the transmission was completed, the software calculated actual throughput (separately for downlink and uplink transmissions), by dividing the file size by the transmission duration measured. The 3G/LTE modem used enabled to obtain some additional system parameters as well, i.e. signal power parameters (RSRP, RSCP) and quality parameters (RSRQ, Ec/Io). Using the ICMP protocol, the ping value could be determined as well. (a)

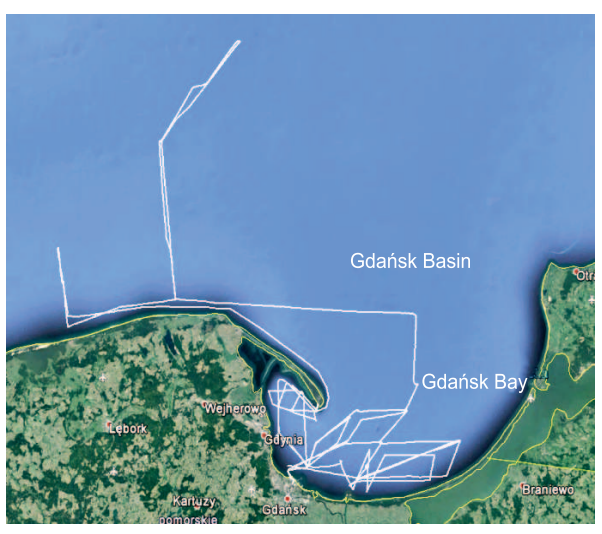

(b)

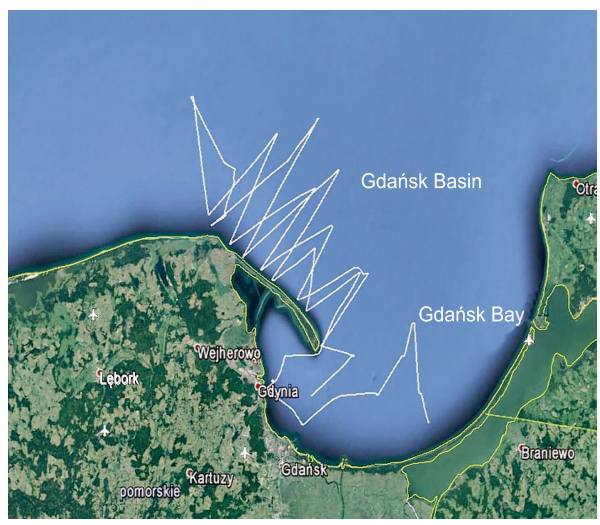

Fig. 3. The ship's routes during the campaigns: (a) in 2015 and (b) in 2017.

${ }^{1}$ LTE USB access head UAH-MC7710-1800-STD, equipped with the Sierra Wireless AirPrime MC7710 LTE/HSPA+ card was used.
The ship that was used during the campaign enabled the antenna to be installed 4 and $10 \mathrm{~m}$ above sea level. That made it possible to model two sizes of a vessel (a "small" ship and a "big" ship). The antenna used during the measurements was of the Apex Magforce MB.TG30.A.305111. It supports the most important bands used by cellular 3G/4G systems, namely 698-960 MHz, 1575.42 MHz and 1710-2700 MHz.

In Fig. 3, the ship's routes during the two measurement campaigns (in 2015 and 2017) are presented.

\section{LQI Parameter}

The LQI parameter grades the quality of the wireless radio links and can be used to compare the quality of various systems, as well as to select the system that is most suitable (i.e. "the best") for a given service/application.

The measurement results obtained on the Baltic Sea during the campaign [5], [6] were used to perform a thorough analysis of the parameters that may affect transmission quality. It was established that in the case of $3 \mathrm{G}$ and LTE networks in zone A, quality is best reflected by the data rate and the received signal power. Other parameters, such as transmission delay, jitter or packet loss ratio, also exert some influence on quality, but the instants when their actual values were significantly different from their mean values were usually observed for low data rates, in areas close to the network's coverage border.

While defining the LQI parameter, it was decided that its value would be in the 0-255 range, representing the system's quality. The following initial assumptions have been made in that respect:

- 0-5 Mbps: LQI < 150,

- 5-10 Mbps: $150<\mathrm{LQI}<220$,

- 10-15 Mbps: $220<\mathrm{LQI}<255$,

- over 15 Mbps: LQI = 255 .

The rationale adopted is that low data rates are most crucial for e-navigation and, consequently, they need to be modeled with the highest level of precision.

Using the above assumptions, the $f(R)$ function can be defined to illustrate the relation between LQI and data rate $\mathrm{R}$ (in Mbps) (Fig. 4):

$$
f(R)=\left\{\begin{array}{ll}
0.025 R^{3}-1.65 R^{2}+36 R & \text { for } 0 \leq R \leq 15 \\
255 & \text { for } R>15
\end{array} .\right.
$$

The relation introduced in Eq. (1) is compliant with the initial assumptions and ensures the continuity of the $f(R)$ function around the point $R=15 \mathrm{Mbps}$.

As a result, LQI will correspond to the data rate which is available in the system analyzed. Obviously, that value will depend on the present state of the system, and such a state will be evaluated based on the messages broadcast by base stations. Parameters corresponding to the received signal level and to service quality will then be compared 


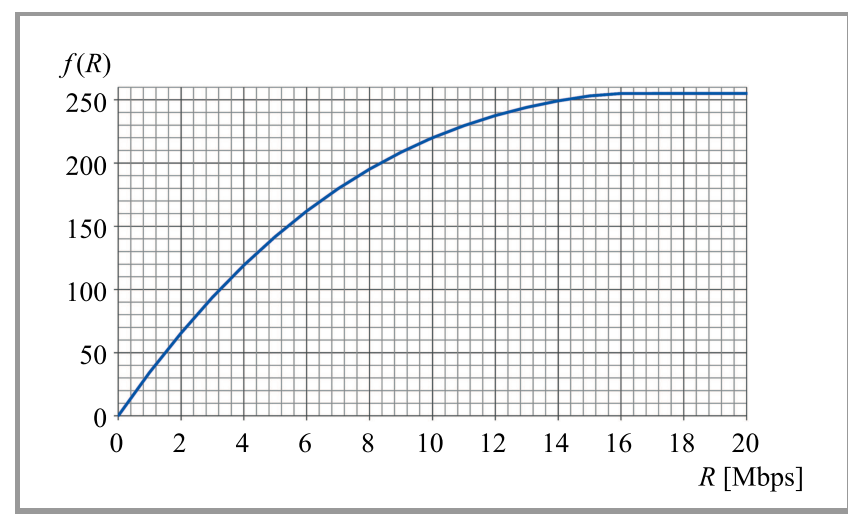

Fig. 4. The $f(R)$ function.

with the measurement data collected during the campaigns, in order to assess the expected system quality and - most importantly - to calculate LQI.

To apply the proposed algorithm, two parameters calculated for the $t$ time need to be known:

- $P_{D}(t)$ - parameter corresponding to the received signal power and the shortest distance from the shore,

- $P_{Q}(t)$ - parameter corresponding to the system quality.

Their values are determined through periodic measurements, but the exact calculation procedure varies depending on the network type ( $3 \mathrm{G}$ vs. LTE). $P_{D}(t)$ and $P_{Q}(t)$ varies in time due to a number of factors (e.g. changes in propagation conditions or network load), so it is necessary to include also (with appropriate weights) the mean values of such factors, as well as their changes over a long period of time. Consequently, the LQI parameter shall be defined in the following way:

$$
L Q I(t)=A \cdot \bar{P}_{D}(t)+B \cdot \bar{P}_{Q}+C \cdot \widetilde{P}_{Q}(t)+D \cdot P_{Q}(t),
$$

where: $A, B, C, D$ - weight coefficients, $\bar{P}_{D}(t)$ - mean value of the parameter corresponding to the received signal power and the distance, $\bar{P}_{Q}(t)$ - mean value of the parameter corresponding to quality, $\widetilde{P}_{Q}(t)$ - change in the quality parameter's mean value observed over time.

Let us assume $\Delta t$ denotes the duration of a single measurement (i.e. the interval time between two consecutive measurement cycles). In that case the period over which the mean values of $P_{D}(t)$ and $P_{Q}(t)$ are calculated can be expressed as $N \cdot \Delta t$, where $N$ is the total number of the collected measurements.

Given the above:

$$
\begin{gathered}
\bar{P}_{D}(t)=\frac{1}{N} \sum_{n=0}^{N-1} P_{D}(t-n \cdot \Delta t), \\
\bar{P}_{Q}(t)=\frac{1}{N} \sum_{n=0}^{N-1} P_{Q}(t-n \cdot \Delta t), \\
\widetilde{P}_{Q}(t)=\bar{P}_{Q}(t)-\bar{P}_{Q}(t-\Delta t) .
\end{gathered}
$$

As per initial assumptions, the LQI value is in the range of 0-255, so whenever the value calculated using Eq. (2) is greater than 255 or lower than 0 , it shall be equal to 255 or 0 , respectively. The weight coefficients A-D might vary for each of the systems analyzed, and the way they are obtained will be explained in the following section.

\section{Calculation of LQI for LTE Cellular Networks}

Once the measurement results have been analyzed, it was established that parameters $P_{Q}(t)$ and $P_{D}(t)$ for the LTE networks should be derived from the Reference Signal Received Quality (RSRQ) and Reference Signal Received Power (RSRP) values [7].

The measurement campaigns indicate that the expected data rate $R$ can be estimated based on the RSRQ level. In Fig. 5, the measured values of the mean RSRQ are presented as a function of the downlink data rates for various frequency bands and channel bandwidths. The curve representing LTE "free mode" serves as a basis for the generic approximation function which will be used to determine the data $R$. The term "free mode" represents a situation where exact frequency parameters of the LTE network are unknown and are selected automatically by the network.

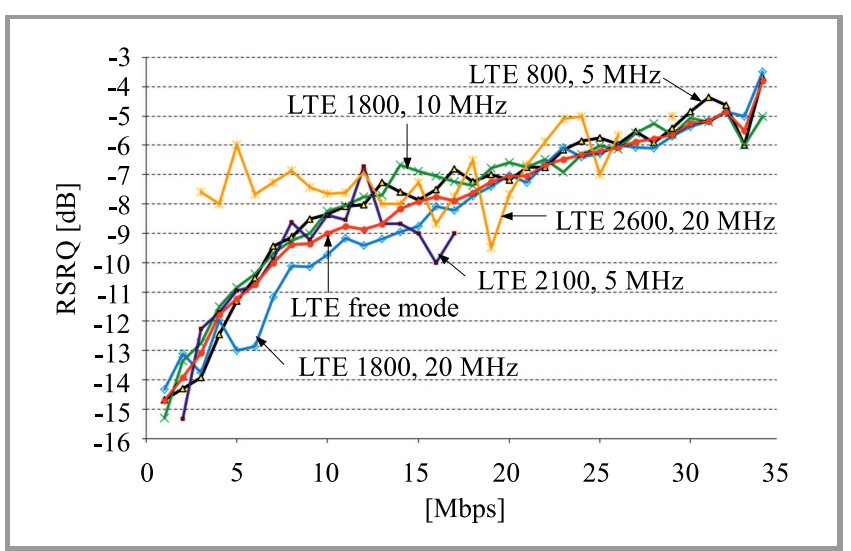

Fig. 5. Mean RSRQ as a function of data rate for different frequency bands and channel bandwidths. (See color pictures online at www.nit.eu/publications/journal-jtit)

For data rates above $15 \mathrm{Mbps}$, the LQI value saturates at 255. The same constraint had to be introduced for the approximation function as well. The relation between the value of the approximation function and RSRQ can be expressed as (Fig. 6):

$$
R[\mathrm{Mbps}]= \begin{cases}10^{\frac{\mathrm{RSRQ}+16.25}{7.1}} & \text { for } \mathrm{RSRQ} \leq-8 \mathrm{~dB} \\ 15 & \text { for } \mathrm{RSRQ}>-8 \mathrm{~dB}\end{cases}
$$

Using the value of the data rate $R$ expected in time $t$, the $P_{Q}(t)$ can be determined on the basis of the $f(R)$ function defined in Eq. (1), i.e.:

$$
P_{Q}(t)=f(R(t))
$$




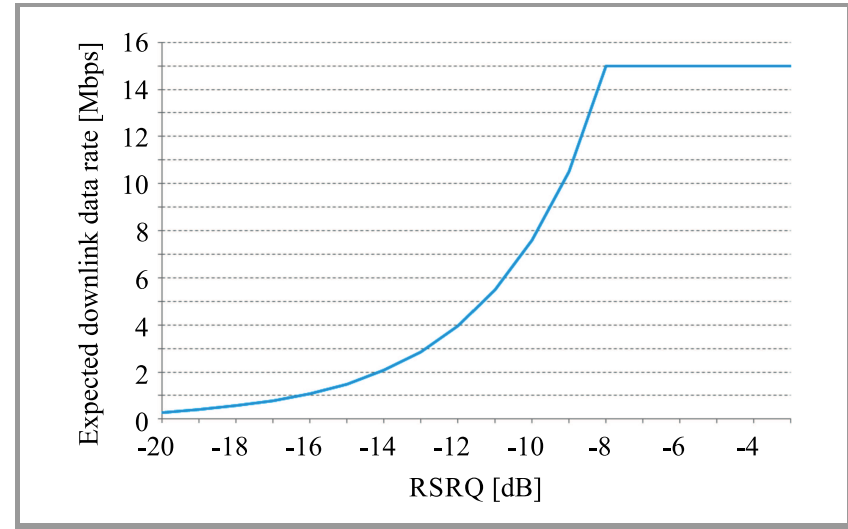

Fig. 6. Approximation function for the LTE networks defined by Eq. (6).

As a result, the $P_{Q}(t)$ will also have its values limited to the $0-255$ range.

In the next step, the $\bar{P}_{Q}(t)$ and $\widetilde{P}_{Q}(t)$ values are calculated using Eqs. (4) and (5), respectively. Having completed that, three out of four parameters required for the LQI evaluation are available.

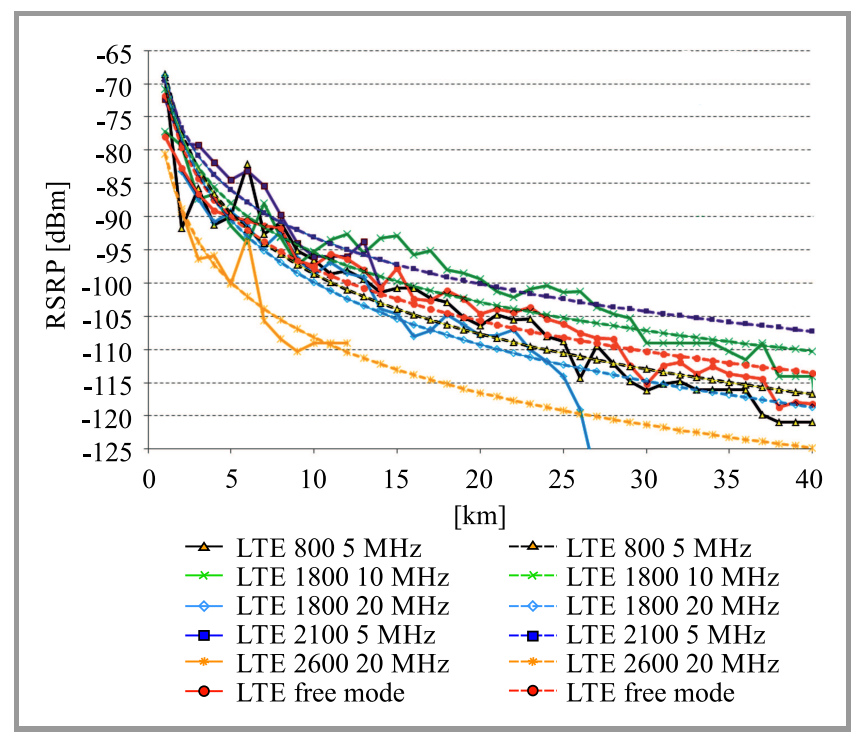

Fig. 7. Relationship between RSRP and the distance from the shore.

The last unknown factor $\bar{P}_{D}(t)$ is related to the received signal power and depends on the distance from the shore. It is calculated using the RSRP. The importance of the $\bar{P}_{D}(t)$ is particularly high in the areas close to the networks' coverage border. Results obtained during the measurement campaigns did not provide a clear and unambiguous relationship between RSRP and the data rate (as it was the case for the RSRQ). On the other hand, a distinct relationship between the RSRP and the distance from the shore has been noticed and it is presented in Fig. 7. The solid lines depict actual measurement results, whereas dotted ones represent the functions that approximate those results.
During the measurement campaigns, it was sometimes the case that the RSRP level and the data rate decreased significantly as the terminal's distance from the shore increased, but at the same time the RSRQ level did not change or even gained a little. The results obtained indicate that in such cases a $5 \mathrm{~dB}$ decrease of RSRP resulted in a mean data rate dropping by approximately $10 \%$. That observation helped establish the relationship between RSRP and the data rate using the following procedure. First, the RSRP level anticipated at a given distance from the shore needs to be calculated using the approximation function shown in Fig. 7. The analytical formulas of these functions are:

$$
\begin{gathered}
\operatorname{RSRP}(d[\mathrm{~km}])[\mathrm{dBm}]= \\
=\left\{\begin{array}{l}
-30.095 \cdot \log (d)-66.50 \text { for LTE } 800,5 \mathrm{MHz} \\
-24.615 \cdot \log (d)-70.81 \text { for LTE } 1800,10 \mathrm{MHz} \\
-31.131 \cdot \log (d)-68.76 \text { for LTE } 1800,20 \mathrm{MHz} \\
-23.509 \cdot \log (d)-69.57 \text { for LTE } 2100,5 \mathrm{MHz} \\
-27.608 \cdot \log (d)-80.58 \text { for LTE } 2600,20 \mathrm{MHz} \\
-26.065 \cdot \log (d)-71.76 \text { for LTE free mode }
\end{array}\right.
\end{gathered}
$$

The approximation functions have been assigned to each of LTE network types analyzed during the measurements (the type is described by network frequency and channel bandwidth). In the next step, the $\operatorname{RSRP}_{\text {approx }}(d)$ value obtained using Eq. (8) compared with the $\operatorname{RSRP}_{\text {meas }}(t)$ value measured at a given time $t$. If the approximated value is greater than the measured one, transmission quality degradation can be expected and the value of $P_{D}(t)$ should be negative. If the opposite is true, one might expect that transmission conditions will improve and, consequently, the $P_{D}(t)$ will assume a positive value. Given the above, the change of the expected data rate can be expressed as:

$$
\Delta R=\frac{\operatorname{RSRP}_{\text {meas }}(t)-\operatorname{RSRP}_{\text {approx }}(d)}{50} \cdot 100 \%
$$

where $\operatorname{RSRP}_{\text {meas }}(t)$ and $\operatorname{RSRP}_{\text {approx }}(d)$ are in $\mathrm{dBm}$.

The measurement results showed also that the RSRP level near the end of the coverage area usually fluctuated between -110 and $-120 \mathrm{dBm}$. The fact that the terminal is getting closer to the border of the network's coverage can be modeled by introducing a new parameter $G_{L T E}$ which will additionally decrease the expected data rate by 10,20 or $30 \%$ :

$$
\begin{gathered}
\quad G_{\text {LTE }}[\%]= \\
=\left\{\begin{array}{ll}
0 & \text { for }-110 \mathrm{dBm}<\mathrm{RSRP}_{\text {meas }}(t) \\
10 \% & \text { for }-115 \mathrm{dBm} \leq \mathrm{RSRP}_{\text {meas }}(t)<-110 \mathrm{dBm} \\
20 \% & \text { for }-120 \mathrm{dBm} \leq \mathrm{RSRP}_{\text {meas }}(t)<-115 \mathrm{dBm} \\
30 \% & \text { for } \mathrm{RSRP}_{\text {meas }}(t) \leq-120 \mathrm{dBm}
\end{array} .\right.
\end{gathered}
$$

Now $P_{D}(t)$ can be defined as:

$$
P_{D}(t)=f\left(R(t) \cdot\left(1+\Delta R-G_{L T E}\right)\right)-f(R(t)) .
$$


In the subsequent step, the value of $\bar{P}_{D}(t)$ can be calculated by applying Eq. (3). Hence, all four parameters necessary to obtain the LQI for the LTE network in zone A are now known and available for further processing.

\section{Calculation of LQI for 3G Cellular Networks}

On the basis of the measurement results, it was established that parameters $P_{Q}(t)$ and $P_{D}(t)$ for UMTS networks should be derived using the values of the Received Signal Code Power (RSCP) and Ec/Io [8], respectively.

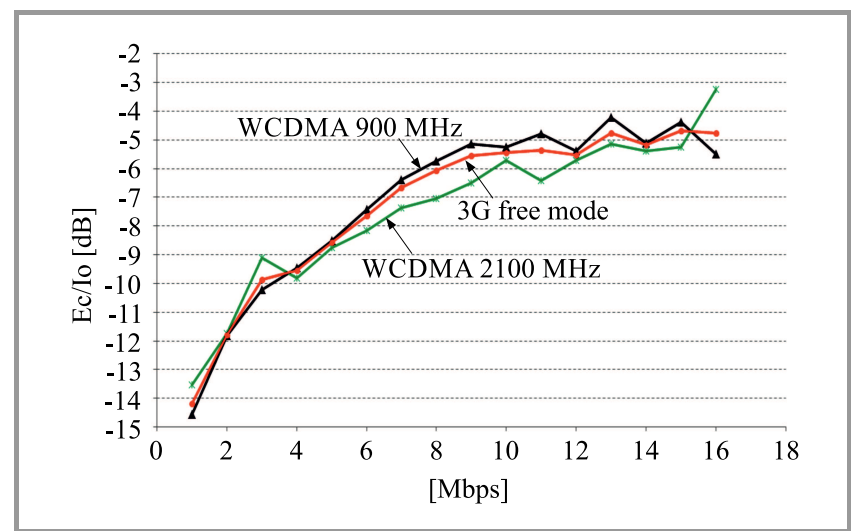

Fig. 8. Relationship between Ec/Io and the downlink data rate.

Similarly to the LTE network, the expected UMTS data rate $R$ can be determined from the measurement data. The relationship between the mean value of Ec/Io and the downlink data rate is presented in Fig. 8 for two frequency bands (900 and $2100 \mathrm{MHz}$ ). As one can observe, those characteristics are relatively similar, so the approximation function was defined on the basis of the $3 \mathrm{G}$ free-mode characteristic. This will make it possible to determine the expected value of the data rate $R$. Just as it was the case for LTE, the $3 \mathrm{G}$ approximation function saturates for data rates

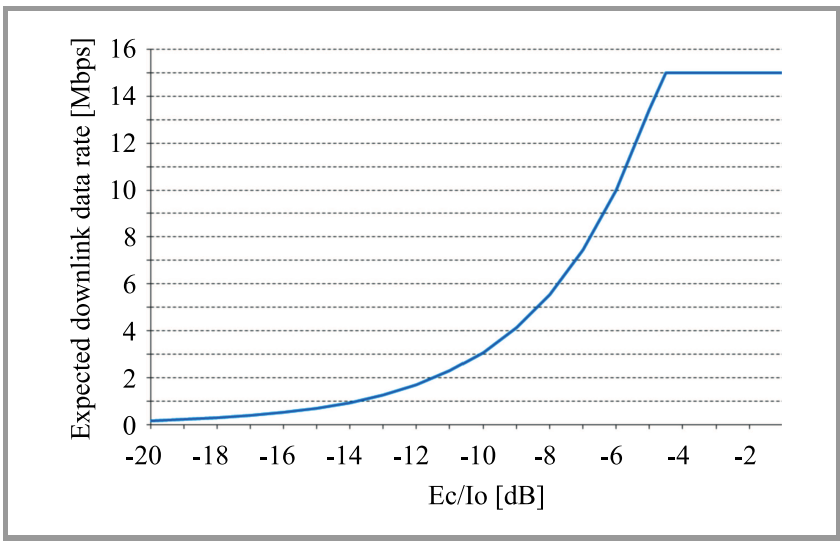

Fig. 9. Approximation function characteristics for the $3 \mathrm{G}$ networks, defined by Eq. (12).

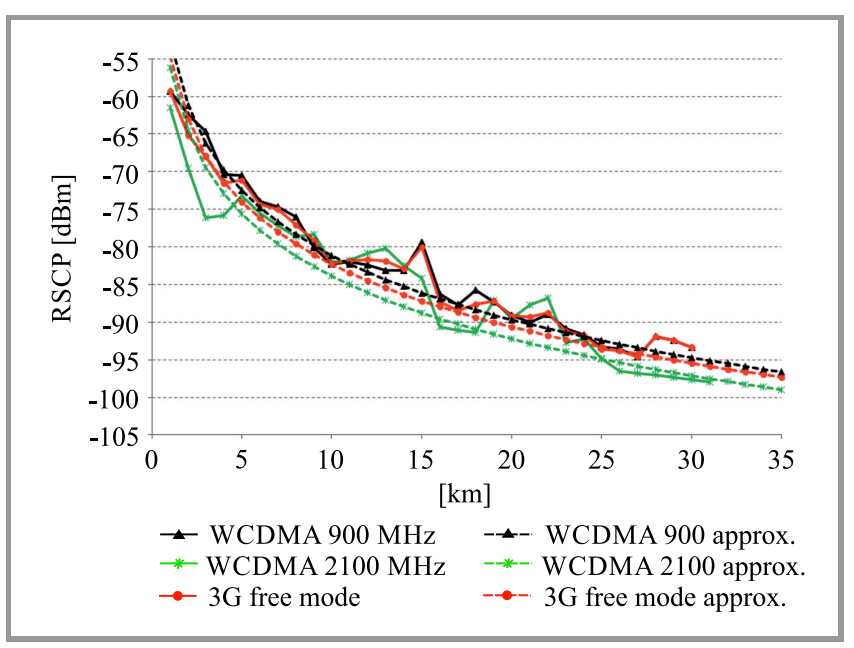

Fig. 10. Relationship between RSCP and the distance from the shore.

above $15 \mathrm{Mbps}$, and its analytical formula can be expressed as (Fig. 9):

$$
R[\mathrm{Mbps}]= \begin{cases}10^{\frac{\mathrm{Ec} / \mathrm{Io}+13.8}{7.8}} & \text { for } \mathrm{Ec} / \mathrm{Io} \leq-4.5 \mathrm{~dB} \\ 15 & \text { for } \mathrm{Ec} / \mathrm{Io}>-4.5 \mathrm{~dB}\end{cases}
$$

Having calculated the value of the data rate predicted for time $t$, it is now possible to calculate $P_{Q}(t)$ using Eq. (7). As a result, $P_{Q}(t)$ is limited to $0-255$.

Next, the $\bar{P}_{Q}(t)$ and $\widetilde{P}_{Q}(t)$ are calculated using Eqs. (4) and (5), respectively.

The last unknown parameter $\bar{P}_{D}(t)$ is related to the received signal power and depends on the distance from the shore. It is calculated using the RSCP. The importance of $\bar{P}_{D}(t)$ is particularly vital in areas close to the coverage border. The measurement did not provide a clear and unambiguous relationship between RSCP and data rate (as it was the case for Ec/Io). On the other hand, a distinct relationship between RSCP and distance from the shore has been observed and is presented in Fig. 10, where solid lines depict measurement results, and dotted ones represent functions that approximate those results. The analytical formulas of these functions are:

$$
\begin{gathered}
\operatorname{RSCP}(d[\mathrm{~km}])[\mathrm{dBm}]= \\
=\left\{\begin{array}{ll}
-28.5 \cdot \log (d)-52.6 & \text { for WCDMA } 900 \\
-27.7 \cdot \log (d)-56.2 & \text { for WCDMA } 2100 \\
-27.6 \cdot \log (d)-54.7 & \text { for 3G free mode }
\end{array} .\right.
\end{gathered}
$$

The approximation functions have been assigned to each of the $3 \mathrm{G}$ network types analyzed during the measurement (the type is described by the frequency utilized by a given $3 \mathrm{G}$ network). In the next step, the $\operatorname{RSCP}_{\text {approx }}(d)$ value obtained using Eq. (13) is compared with the $\operatorname{RSCP}_{\text {meas }}(t)$ value measured in time $t$. If the approximated value is greater than the measured one, transmission quality degradation can be expected and the value of $P_{D}(t)$ should be negative. If the opposite is true, one may expect that 
transmission conditions will improve and, consequently, $P_{D}(t)$ will be assume a positive value. Given the above, the change in the expected data rate can be expressed as:

$$
\Delta R=\frac{\operatorname{RSCP}_{\text {meas }}(t)-\mathrm{RSCP}_{\text {approx }}(d)}{50} \cdot 100 \%,
$$

where $\operatorname{RSCP}_{\text {meas }}(t)$ and $\operatorname{RSCP}_{\text {approx }}(d)$ are in $\mathrm{dBm}$.

The measurements have also shown that the RSCP level near the coverage border usually fluctuated between -100 and $-120 \mathrm{dBm}$. The fact that the terminal is getting closer to the network's coverage border can be modeled by introducing a new parameter, $G_{3 \mathrm{G}}$, which will additionally decrease the expected data rate by 10,20 or $30 \%$, according to the formula:

$$
\begin{gathered}
\quad G_{3 \mathrm{G}}[\%]= \\
=\left\{\begin{array}{ll}
0 & \text { for }-90 \mathrm{dBm}<\mathrm{RSCP}_{\text {meas }}(t) \\
10 \% & \text { for }-105 \mathrm{dBm} \leq \mathrm{RSCP}_{\text {meas }}(t)<-90 \mathrm{dBm} \\
20 \% & \text { for }-120 \mathrm{dBm} \leq \mathrm{RSCP}_{\text {meas }}(t)<-105 \mathrm{dBm} \\
30 \% & \text { for } \mathrm{RSCP}_{\text {meas }}(t) \leq-120 \mathrm{dBm}
\end{array} .\right.
\end{gathered}
$$

The $P_{D}(t)$ can now be defined as:

$$
P_{D}(t)=f\left(R(t) \cdot\left(1+\Delta R-G_{3 \mathrm{G}}\right)\right)-f(R(t)) .
$$

In the final step, the $\bar{P}_{D}(t)$ can be calculated by using Eq. (3).

\section{Calculation of LQI Weight Coefficients}

Using research presented in Sections 4 and 5, a more thorough analysis of the proposed algorithm has been performed based on the following assumptions:

- weight coefficients that appear in Eq. (2) will be calculated independently for $3 \mathrm{G}$ and LTE networks;

- analysis will cover downlink transmission only;

- three different averaging periods for $P_{Q}$ and $P_{D}$ parameters have been adopted (3,5 and $10 \mathrm{~min})$;

- the measurement series lasting at least $15 \mathrm{~min}$ have been extracted from the measurement data. Those series account for approximately $90 \%$ of all the data obtained during the campaigns;

- some data was discarded before further analysis. That includes those pieces of data for which the relationship between RSRQ (Ec/Io) and the data rate varied significantly from the overall relationship observed throughout the entire campaign. Once this step has been performed, the data left for further analysis comprised approximately $80 \%$ of the initial data set.

Next, the following elements were calculated for each of the measurement series extracted:
- three values of the LQI corresponding to three different averaging periods, denoted as LQI 3/5/10;

- based on the measurements, the LQI value corresponding to a given data rate was calculated: LQI $\left(R_{\text {current }}\right)=f\left(R_{\text {current }}\right)$, and compared with the expected LQI 3/5/10 calculated using the system parameters;

- for each measurement point collected, the average error of LQI calculation was determined: $\Delta \mathrm{LQI}=$ LQI 3/5/10 - LQI $\left(R_{\text {current }}\right)$;

- for each measurement series, the average error $\Delta \mathrm{LQI}$ and its standard deviation were calculated;

- for all the series, the weighted average value and average standard deviation were calculated.

Coefficients A-D from Eq. (2) were derived empirically, in such a way that the average error of LQI calculation was equal to zero and the value of its standard deviation was kept as low as possible. During the first stage of the LQI algorithm verification, it was proven that this method models the actual performance of LTE/3G networks quite faithfully, and that the best results can be achieved for a 3-minute averaging period. Those observations served as a starting point for further activities. The analysis conducted has also shown that quality parameters of cellular networks should be monitored with a higher frequency (at that point such measurements were taken every $30 \mathrm{~s}$ ). As a result, before further analysis, it was necessary to introduce some modifications to the measurement procedures implemented in the Mobile Measurement Platform [4].

During the second measurement campaign, the 3-second interval was used, which significantly increased the amount of the data collected. The way that data was analyzed was quite similar to the method adopted in the first step of the verification process. However, the averaging period for $P_{D}(t)$ and $P_{Q}(t)$ was 3 minutes, and the duration of the data series that was taken into account had to exceed 5 minutes.

The most significant difference between the first and the second verification was that in the latter we did not compare the $\Delta \mathrm{LQI}$ error, but the difference between the expected data rate derived from LQI and the actual (real) data rate observed at a given time. Additionally, it was assumed that whenever the real data rate exceeded $15 \mathrm{Mbps}$, the value used for the purpose of comparison was equal to $15 \mathrm{Mbps}$, which results from the adopted definitions of LQI and the $f(R)$ function.

Having completed this entire procedure, the final formulas defining the LQI for 3G and LTE, respectively (which take into account the empirical coefficients A-D), are:

$$
\begin{gathered}
L Q I_{3 \mathrm{G}}(t)=0.57 \cdot \bar{P}_{D}(t)+0.95 \cdot \bar{P}_{Q}(t)+ \\
+0.56 \cdot \widetilde{P}_{Q}(t)+0.14 \cdot P_{Q}(t) \\
L Q I_{L T E}(t)=0.53 \cdot \bar{P}_{D}(t)+0.89 \cdot \bar{P}_{Q}(t)+ \\
+0.50 \cdot \widetilde{P}_{Q}(t)+0.12 \cdot P_{Q}(t) .
\end{gathered}
$$


In both cases the average error of the data rate estimation was equal to zero, and the standard deviation was $1.8 \mathrm{Mbps}$ for the $3 \mathrm{G}$ network and $3.7 \mathrm{Mbps}$ for LTE.

\section{Conclusions}

Versatility is one of the unquestionable advantages of the method introduced in this paper. The algorithm is not based on a single factor, but rather takes into account both power and quality factors. To a certain extent, it can also be adapted and tailored for the purpose of assessment of other links (e.g. WiMAX). It should be underlined that the correct operation of the algorithm has been verified and confirmed in the course of an extended measurement campaign that was performed in the target environment - i.e. at sea. On the other hand, it has to be strongly stated that the values on which the algorithm is based - power and quality as important as they are, are not the only factors that affect the actual quality of a radio link. The final output of the LQI algorithm is hence an approximation of the real value. Another limitation of the method is the fact that due to the averaging process, quality estimation is slightly delayed. An attempt has been made to mitigate that issue by using an additional power-related parameter which enables to "predict" that the terminal is approaching the cell's border.

In general, the LQI algorithm proposed is applicable mostly to the systems for which the measurable quality and power parameters have been defined, i.e. 3G/HSPA and LTE/LTEA cellular networks or WiMAX networks. The algorithm has been verified both in the coastal waters (the Bay of Gdańsk) and at open sea (see Fig. 3). Basically, the method can be utilized for any type of shore-based infrastructure and for any telecommunications providers, but in the latter case the specific features of their networks should be taken into account. It has to be underlined that the measurements described in this paper were conducted for two different cellular operators.

Obviously, besides the systems for which the LQI method is applicable, there exist other communication solutions that might be utilized in the netBaltic network as well. That is particularly the case in zone $\mathrm{B}$, for which Wi-Fi communication is likely to be the major transmission mechanism. As opposed to cellular systems, Wi-Fi networks do not offer any unambiguous quality parameters and their characteristics are very different from 3G/LTE (e.g. small distances between stations, low antenna heights at both radio link ends, license-free ISM band utilization, etc.). In light of the above, an attempt has been made to define a universal quality parameter for the netBaltic zone B, which would be based on the measured intervals between consecutive control packets. Those packets will be introduced in the netBaltic network and they will be primarily used to maintain the network's topology.

It should be noted that, to a certain extent, the LQI algorithm presented in this paper can be considered a special type of cognitive radio (CR). In CR, the frequency channel is selected automatically, depending on its availability, to avoid congestion and interference with primary users. In the case of the LQI algorithm, this "automatic selection" refers not only to the frequency, but most notably to the standard of wireless transmission. So, here, the primary goal is to ensure the best possible transmission conditions for the user (by maximizing the LQI metric). On the other hand, it can also be stated that in the case of CR, the algorithm is relatively simpler, because the same measure can be used to achieve the goal (i.e. to select the optimum frequency channel), whereas in the LQI algorithm, the selection is made from among several various, incomparable transmission standards. Consequently, in this case, the same measure cannot be used for LTE, 3G, Wi-Fi or any other system discussed. That is why the authors provided, in the paper, two separate LQI formulas for LTE and 3G see Eqs. (17) and (18). This accentuates the fact that on the conceptual level, the LQI algorithm is rather complex and requires analysis of many input parameters which vary in time.

Even though this is beyond the scope of this article, it should be also noted that the range (service coverage) of radio systems in zone $\mathrm{A}$ is generally limited. Currently, organizations such as IALA and ITU are working on a novel maritime communication standard known as VHF Data Exchange System (VDES) [9], [10] which should substantially help overcome that limitation. Another factor that will strongly contribute to the enhancement of quality of maritime communication is e-navigation. This concept has been developing rapidly over the past years, which is demonstrated by a large number of relevant publications and projects and by a general interest shown by the maritime community (including IMO and IALA). One of the projects dealing with that area is EfficienSea 2.0 [11], in which the National Institute of Telecommunications is deeply involved.

\section{Acknowledgments}

This work has been partially supported by the (Polish) Applied Research Program under the Grant No. ID PBS3/ A3/20/2015, founded by the National Center for Research and Development.

\section{References}

[1] netBaltic project website (in Polish) [Online]. Available: www.netbaltic.pl (accessed: 29 Jan. 2018).

[2] Z. Jinglong, M. Jacobsson, E. Onur, and I. Niemegeers, "A novel link quality assessment method for mobile multi-rate multi-hop wireless networks", in Proc. 6th IEEE Consumer Commun. and Netw. Conf. CCNC 2009, Las Vegas, NV, USA, 2009

(doi: 10.1109/CCNC.2009.4784751).

[3] B. C. YI, X. Jin, C. Liu, X. Li, and Y. Wei, "Relative link quality assessment and hybrid routing scheme for wireless mesh networks", in Proc. IEEE Int. Conf. on Commun. ICC 2011, Kyoto, Japan, 2011 (doi: 10.1109/icc.2011.5963284). 
[4] K. Bronk, R. Niski, and B. Wereszko, "Measurement of data transmission quality in the area of Tri-City in the networks of selected cellular operators", Przegląd Telekomun. + Wiadomości Telekomun., no. 4, 2015, pp. 197-200 (in Polish).

[5] Report from the measurement campaign on the Baltic Sea, 2015, netBaltic project documentation, National Institute of Telecommunications.

[6] Report from the measurement campaign on the Baltic Sea, 2017, netBaltic project documentation, National Institute of Telecommunications.

[7] 3GPP TS 36.214 V14.2.0 (2017) Technical Specification Group Radio Access Network; Evolved Universal Terrestrial Radio Access (E-UTRA); Physical layer; Measurements (Release 14).

[8] 3GPP TS 25.215 V14.0.0 (2017) Technical Specification Group Radio Access Network; Physical layer; Measurements (FDD) (Release 14).

[9] ITU-R M.2092-0 (10.2015) "Technical characteristics for a VHF data exchange system in the VHF maritime mobile band".

[10] K. Bronk, M. Mazurowski, D. Rutkowski, and B. Wereszko, "Badania symulacyjne warstwy fizycznej naziemnego segmentu systemu VDES (Simulation analysis of the physical layer of the VDES terrestrial segment)", Przegląd Telekomun. + Wiadomości Telekomun., no. 6, 2016, pp. 467-470 (in Polish).

[11] K. Bronk, A. Lipka, R. Niski, B. Wereszko, and K. Wereszko, "Hybrid communication network for the purpose of maritime applications", Int. J. of Maritime Engin., vol. 159, part A1, pp. 115-122, 2017 (doi: 10.3940/rina.ijmme.2017.a1.396tn).

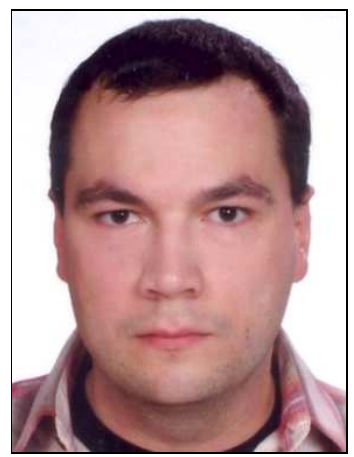

Krzysztof Bronk holds a Ph.D. degree (2010) and is an Assistant Professor at the National Institute of Telecommunications. He is an author or co-author of more than 60 reviewed scientific articles and publications, technical documents and studies. His research focuses mainly on radio communication systems, networks design and planning, development of software-defined and cognitive radio systems, multi-antenna technology, cryptography, propagation analysis, transmission and coding techniques, as well as positioning systems and techniques. His interests include also multi-thread and object-oriented applications, devices controlling applications, DSP algorithms and quality measurement solutions.

ORCID ID: https://orcid.org/0000-0002-3594-8462

E-mail: K.Bronk@itl.waw.pl

National Institute of Telecommunications

Wireless Systems and Networks Department

Jaśkowa Dolina 15

80-252 Gdańsk, Poland

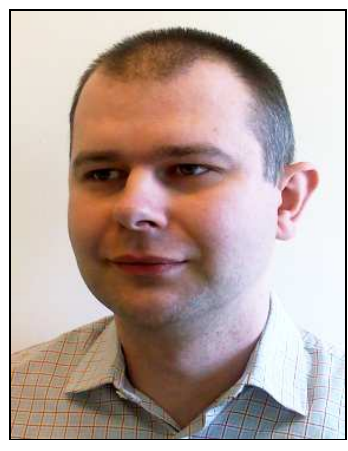

Adam Lipka received his M.Sc. and Ph.D. degrees in Telecommunications from the Gdańsk University of Technology in October 2005 and June 2013, respectively. Since January 2006, he has been working at the National Institute of Telecommunications at its Wireless Systems and Networks Department in Gdańsk (currently as an Assistant Professor). His scientific interests include contemporary transmission techniques, MIMO systems and radio waves propagation. He is an author or co-author of over 50 scientific papers and publications.

ORCID ID: https://orcid.org/0000-0002-2919-4270

E-mail: A.Lipka@itl.waw.pl

National Institute of Telecommunications

Wireless Systems and Networks Department

Jaśkowa Dolina 15

80-252 Gdańsk, Poland

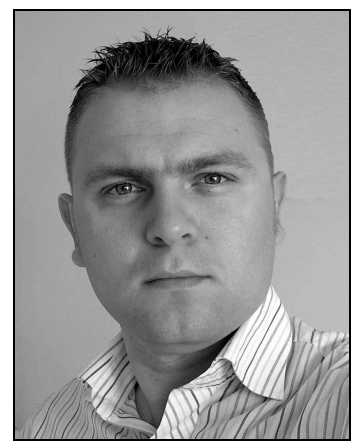

Rafał Niski graduated from Gdańsk University of Technology in 2001, receiving an M.Sc. in radio communications. Since then, he has been working at the National Institute of Telecommunications in Gdańsk, at first as an Assistant Professor, and after receiving his Ph.D. degree in 2006, as an Associate Professor. Between 2005 and 2012 he was the Head of Wireless Systems and Networks Department, and since 2016 he has been the Head of the Network and Equipment Measurement section. His scientific research concerns the theory and techniques of mobile communication, radio networks design and planning, as well as measurements of transmission and quality-related parameters in radio networks. $\mathrm{He}$ is an author or co-author of nearly 90 scientific publications. Since 2007 he has been a member of the Scientific Council of the National Institute of Telecommunications.

ORCID ID: https://orcid.org/0000-0002-5106-9046

E-mail: R.Niski@itl.waw.pl

National Institute of Telecommunications

Wireless Systems and Networks Department

Jaśkowa Dolina 15

80-252 Gdańsk, Poland 